\title{
Attaque et défense de l'intestin : trois facteurs protéiques entrent en scène!
}

L'intestin, lieu d'accès privilégié des microorganismes, constitue une barrière efficace contre leur entrée dans l'organisme, en mettant en jeu une défense passive, principalement liée à la présence de mucus, et une défense active, par la synthèse d'agents neutralisants spécifiques [1, 2]. Parmi ces micro-organismes, les rotavirus, causent annuellement, à l'échelle mondiale, jusqu'à 130 millions de cas de gastro-entérites et diarrhées et sont responsables de la mort de près d'un million d'enfants dans les pays en voie de développement. Ces chiffres, à eux seuls, justifient toute étude visant à comprendre le mécanisme d'action des rotavirus, et à élucider les systèmes biologiques de défense contre les micro-organismes. En ce qui concerne l'attaque, le facteur responsable des diarrhées provoquées par les rotavirus, vient d'être identifié comme étant la glycoprotéine virale non structurale, NSP4 [3]. Dans le camp de la défense, la démonstration in vivo du rôle majeur joué par les immunoglobulines sécrétoires IgA, et l'identification, chez l'homme, de deux gènes codant pour des protéines «défensives» de la famille des défensines $[4,5]$, viennent, une nouvelle fois, confirmer l'importance de la composante active de la fonction de défense intestinale. C'est, incidemment, au cours de la préparation d'anticorps contre la protéine NSP4 du rotavirus, que cette protéine s'est révélée capable, à elle-seule, ris. L'effet diarrhéique est observé une heure après l'administration intrapéritonéale de la protéine recombinante purifiée, et persiste pendant au moins 24 heures. Si la réponse intestinale varie avec la concentration de la protéine et le mode d'administration, elle dépend surtout étroitement de l'âge de l'animal. Ainsi, après l'injection intrapéritonéale d'une nanomole de NSP4, des diarrhées sont observées chez $100 \%$ des souris de $6-7$ jours et $60 \%$ des souris de 8-9 jours. L'administration intra-iléale de 0,5 nanomole de la protéine est plus efficace et affecte $100 \%$ des souris de $8-9$ jours, les souris de 17 jours restant insensibles. A tous les âges, la protéine administrée par injection intramusculaire est sans effet. Le peptide synthétique de 22 acides aminés, NSP4 114-135, qui mime les effets spécifiques de la protéine recombinante, montre aussi une plus grande efficacité chez les jeunes animaux et lors de l'administration par voie intrailéale. La tyrosine centrale (position 131) et les 10 acides aminés de la région aminoterminale sont particulièrement déterminants pour l'activité $\mathrm{du}$ peptide. Les anticorps dirigés contre NSP4 114-135 sont puissants puisque, en inoculation intrapéritonéale «préventive», ils neutralisent l'effet diarrhéique du peptide. En outre, les souris nées de mères immunisées contre NSP4 114-135 ou traitées oralement par l'anticorps anti-NSP4, sont protégées d'une infection par le rotavirus infectieux
SA11 (simian agent 11). L'action du NSP4, qui se traduit par une augmentation des sécrétions hydroélectrolytiques sans altérations histologiques de la muqueuse intestinale, rappelle singulièrement le mécanisme d'action des entérotoxines bactériennes. En effet, en mobilisant le calcium intracellulaire, NSP4 augmente, in vitro, la sécrétion des ions chlorures par la muqueuse iléale, et potentialise l'effet de la forskoline, stimulant de l'adénylyl cyclase. Ici aussi, la sensibilité de la réponse au peptide diminue fortement avec l'âge des animaux. Pour expliquer cette relation de dépendance entre les symptômes diarrhéiques et l'âge, un modèle a été proposé : qui implique l'existence, au niveau de la muqueuse intestinale, de deux récepteurs spécifiques du rotavirus, un premier récepteur reconnaissant la particule virale et permettant son entrée dans la cellule, sa réplication et, par conséquent, la synthèse et la sécrétion de la protéine NSP4 dans la lumière intestinale ; un deuxième récepteur synthétisé par les cellules adjacentes et reconnaissant la protéine NSP4 sécrétée. La présence de particules virales dans les selles de souris diarrhéiques et non diarrhéiques rend ce modèle tout à fait cohérent et la perte de récepteurs de NSP4 ou/et des canaux chlorures sensibles au calcium chez les souris âgées pourrait être responsable de l'insensibilité des animaux âgés à la protéine NSP4. On comprend aujourd'hui d'autant mieux l'identi- 
fication de la protéine NSP4 en tant qu'entérotoxine virale et la redéfinition du gène NSP4 comme "gène de virulence».

Une composante active de la défense intestinale contre les micro-organismes, et notamment les rotavirus, implique les anticorps sécrétoires IgA, libérés en quantités importantes par les lymphocytes au niveau de la sous-muqueuse intestinale [6]. Alors que, in vitro, les IgA oligomériques agissent en formant des complexes avec les virus ayant pénétré les cellules intestinales et bloquent ainsi leur réplication, le mécanisme de défense opérant in vivo reste énigmatique. En effet, alors que la présence d'anticorps sériques contre le rotavirus n'est pas toujours associée à un effet protecteur, une étroite corrélation entre la quantité d'IgA intestinales spécifiques du rotavirus et un effet antiviral semble exister in vivo. Une équipe de Californie vient d'apporter des bases moléculaires solides attestant in vivo la participation des IgA secrétoires dans la protection contre les rotavirus [4]. Ainsi, des cellules d'hybridomes synthétisant des anticorps monoclonaux d'isotypes IgA et IgG, dirigés contre une protéine majoritaire des rotavirus, la protéine VP6, et l'hémagglutinine virale VP4, sont injectées par voie sous-cutanée à des souris histocompatibles infectées deux semaines plus tard par le rotavirus murin (souche EC). Il apparaît que seules les souris transplantées avec l'hybridome synthétisant des IgA anti-VP6 (anticorps non neutralisant in vitro) sont protégées de l'infection. Les IgG anti-VP6, et les IgA anti-VP4 (qui ont une activité neutralisante in vitro), n'ont, paradoxalement, aucun effet protecteur contre l'infection virale. Cet effet protecteur des IgA anti-VP6 est d'autant plus intéressant qu'il se manifeste également contre d'autres souches de rotavirus comme le rotavirus de type EW. Le fait que les IgA d'hybridomes soient retrouvées dans les selles de toutes les souris indique un acheminement correct à la membrane intestinale et la mise en jeu d'un mécanisme bien connu de transcytose. L'administration intrapéritonéale de liquide d'ascite contenant les IgA anti-VP6 diminue fortement les symptômes diarrhéiques, alors que l'administration orale ou luminale directe n'a aucun effet protecteur contre le virus. Ces travaux indiquent clairement que l'effet antiviral des IgA nécessite une transcytose plutôt qu'un événement extracellulaire dans la lumière digestive, phénomène qui pourrait, en particulier, expliquer l'inefficacité des IgG, qui n'accèdent pas à la lumière intestinale, contrairement aux IgA secrétoires. Ainsi, la démonstration de l'effet protecteur des IgA anti-VP6 contre plusieurs souches virales et l'inefficacité de l'administration orale de ces molécules constituent aujourd'hui des informations capitales pour l'élaboration de vaccins contre les rotavirus.

La grande sensibilité aux agents pathogènes des organismes jeunes, exacerbée chez les nouveau-nés prématurés, témoigne sans aucun doute d'une immaturité du système de défense intestinale. Une démonstration convaincante de cette situation est apportée par une étude angloaméricaine identifiant, chez l'homme, deux gènes codant pour des défensines [5]. Ces peptides font partie d'une des familles, la mieux connue, de peptides antimicrobiens synthétisés et sécrétés par des cellules myéloïdes et non myéloïdes comme les cellules intestinales de Paneth $\left(\mathrm{m} / \mathrm{s} n^{\circ} 4\right.$, vol. 12, p. 517) [2]. Ces peptides cationiques sont constitués de 30 à 35 acides aminés et sont caractérisés par un motif cystéine très conservé. Ils exercent leur action défensive en perméabilisant la membrane externe et interne de bactéries, champignons et certains virus. $\mathrm{Si}$, chez la souris, on a dénombré jusqu'à 16 gènes distincts, chez l'homme, deux gènes codant pour des défensines, $H D-5$ et $H D-6$, viennent d'être identifiés. De façon tout à fait remarquable, l'expression de ces gènes est modulée au cours du développement, le gène $H D-5$ étant exprimé à 13,5 semaines de gestation dans l'intestin grêle et le côlon alors qu'à 17 semaines, seul l'intestin grêle synthétise le peptide. Le gène HD-6, quant à lui, est exprimé et à partir de 13,5-17 semaines de gestation, dans l'intestin grêle unique- ment. Ce profil d'expression coïncide avec l'apparition de cellules de Paneth au niveau de l'épithélium intestinal, suggérant ainsi que la défensine pourrait être un marqueur précoce de la différentiation de ce type de cellule intestinale. L'importance de ces peptides dans la défense de l'organisme contre les microorganismes, est illustrée par le fait que l'expression des gènes $H D-5$ et HD-6, chez le fotus de 24 semaines, est 40 à 250 fois plus faible que chez l'adulte, l'expression du gène $H D-5$ étant toujours supérieure à celle du gène $H D-6$. Alors que le rôle précis des défensines dans la défense de l'organisme contre les micro-organismes est encore mal défini, les hypothèses avancées proposent que ces peptides ont un rôle limitant sur la prolifération de la flore intraluminale et empêchent le passage des bactéries à travers la barrière intestinale. Quoiqu'il en soit, ces peptides méritent l'intérêt qu'on leur porte dans la mesure où leur faible expression chez le fotus humain est probablement une des causes de la prédisposition des nouveau-nés prématurés à l'infection par les micro-organismes intestinaux, l'entérocolite nécrosante étant une forme gravissime fréquemment rencontrée chez ces nourrissons.

B.A.

1. Pattus F. Les armes peptidiques de la défense antimicrobienne: un champ d'investigation en pleine expansion. médecine/sciences $1992 ; 8$ : 420-2.

2. Nicolas P, Mor A, Delfour A. Les peptides de la défense antimicrobienne des vertébrés. médecine/sciences $1992 ; 8$ : 423-31.

3. Ball JM, Tian P, Zeng C, Porris AP, Estes MK. Age-dependent diarrhea induced by a rotaviral nonstructural glycoprotein. Science 1996; 272 : 101-4.

4. Burns JW, Siadat-Pajouh M, Krishnaney AA Greenberg HB. Protective effect of rotavirus VP6specific IgA monoclonal antibodies that lack neutralizing activity. Science 1996 ; 272 : 104-7.

5. Mallow EB, Harris A, Salzman N, Russell JP, DeBerardinis RJ, Ruchelli E, Bevins CL. Human enteric defensin. Gene structure and developmental expression. I Biol Chem 1996; 271 : 4038-45.

6. Butor C, Couëdel-Courteille A, Venet A, Guillet JG. Immunité locale et vaccination. médecine/sciences $1995 ; 11$ : 703-11. 